\title{
DISCUSSION
}

\section{Understanding and using technological affordances: a commentary on Conole and Dyke}

\author{
Tom Boyle* \& John Cook \\ London Metropolitan University, UK
}

\section{Introduction}

The paper by Conole and Dyke sets the context by pointing to a number of problems that inhibit the widespread, effective use of Information and Communication Technologies (ICT) to support learning. They argue that this situation highlights the need to explore a theoretical basis for the use of ICT to support learning. The central argument of the paper is that the notion of affordances can make a significant contribution to this endeavour. The paper aims to articulate the potential impact of these affordances primarily through the development of a taxonomy. It draws on social constructivist theory to help understand and articulate the impact of these affordances.

The concept of affordances is potentially both rich and provocative. Conole and Dyke provide a refreshing and diverse look at the theoretical basis for the use of ICT to support learning. We have structured our commentary around six questions that are provided by Conole and Dyke in their Discussion section. Whilst examining these questions we highlight various issues raised by the paper, which we believe, need further consideration and clarification.

\section{Commentary}

Question 1: How valuable is the concept of affordances and does its application provide any really new insight into the inherent properties of technologies?

There are really two questions here. This section will concentrate on the first question, which involves clarifying the central concept of affordances. This central concept in the paper is only briefly explored before the paper moves to building a taxonomy. McGrenere and Ho (2000) point out, however, that the concept of

\footnotetext{
*Corresponding author: Learning Technology Research Institute, London Metropolitan University, UK. Email: t.boyle@londonmet.ac.uk
} 
affordance is not well understood, and that there is considerable ambiguity and confusion in the use of the term. A brief exploration of how the concept has been used in the psychological and human computer interaction (HCI) literature helps to clarify the important issues surrounding the different uses of this term.

The concept of affordance was developed by J. J. Gibson. Gibson (1977, 1979) argued for an ecological approach to understanding human perception. He argued that the environment and animals have co-evolved. There are features of the environment that afford (i.e. enable) perception and action in that environment. They are not constructed by the person. They exist independently in the environment, and are discovered rather that constructed by the human (or animal) actor. Thus a rigid surface stretching to the horizon under our feet affords locomotion; an object of a certain size affords grasping and so on. Gibson's concept has to be understood within this ecological approach. He argues, 'The world of physical reality does not consist of meaningful things. The world of ecological reality ... does' and 'their meanings can be discovered' (Gibson, 1979, p. 33).

The idea of affordances was popularized in the HCI community by Donald Norman in his book 'The Psychology of Everyday Things' (Norman, 1988). Norman, however, introduced a number of changes, which led to subsequent confusion and variability in the use of the concept. The most fundamental difference relates to Norman's concern, as a designer, with making affordances salient so that users can easily perceive them. Norman conflates two important but different things - designing the utility of an object and designing the way in which that utility is conveyed to the user. In his later work Norman acknowledges the confusion and seeks to distinguish 'real from perceived affordances' (Norman, 1998, p. 123).

McGrenere and Ho (2000) provide an informative analysis to clarify the situation. They distinguish the utility of an object, the actions it affords for the user, from the usability of an object, which is related to the perceptual information that signals the affordances. They propose that there can be degrees of affordance (Warren, 1995), and that affordances can be related in sequential or nested structures. This analysis and clarity in the use of the term is missing in the article by Conole and Dyke which moves rapidly to using the term before analysing and articulating it. The danger is that it falls into the problem identified by McGrenere and Ho, 'As the concept of affordances is used currently, it has marginal value because it lacks specific meaning' (McGrenere \& Ho, 2000, p. 8).

\section{Question 2: How valid is the methodological approach suggested?}

Another important issue is a critical consideration of the 'taxonomic' approach to capturing and representing the implications of the concept. Conole and Dyke argue that practitioners are often unclear about how to use the technology appropriately. They then present a taxonomy of ICT affordances as a contribution to solving this problem. This taxonomy is grounded in the base of 'current social theory and critique'. 
The affordances listed are various. The first is called 'accessibility'-the Internet 'affords' opportunities for accessing information and knowledge in a new way. The authors, however, use accessibility in a non-standard way. They argue for a shift in the primary challenge from finding to selecting relevant information. This diverges from the standard concern with accessibility as 'Access by everyone regardless of disability.' (Web Accessibility Initiative: http://www.w3.org/WAI/).

Another affordance proposed by the authors is 'Speed of change', which refers to attempts to understand the question of how technology can 'be used to enable students to navigate their way through the myriad of changing information and make more informed decisions'. However, it is left unclear how this can act as affordance. A large list of 'affordances' then follows in the paper; which includes diversity, communication and collaboration, reflection, multi-modal and non-linear learning, and so on. Rather than elaborating on how any one of these 'affordances' could be relevant to a learner or a practitioner the authors tend to indulge in a certain amount of hopeful expectation that affordances and abilities will simply emerge; for example in the context of reflective affordances: 'Perhaps new forms of reflection and critique will emerge in response to more transitory and digital text.'

\section{Question 3: What other approaches might be taken?}

The third question points to the theoretical tension introduced by the use of this concept - the slotting into a social constructivist approach ignores the quite different theoretical framework within which Gibson developed the concept of affordances. The concept of affordances arises from an (biological) ecological approach to human cognition and perception. The natural home for an approach based on Gibsonian affordances is not social theory. The concept might have been used to challenge the limits of social theory rather than be simply being assimilated into it.

A key question is-does the concept of affordances really help? The authors articulate certain opportunities that the technology makes available to practitioners. They then balance these positive features with difficulties or challenge that are raised for users. If these barriers are not dealt with effectively then the opportunities may not be exploited. How does the concept of affordance enrich this approach?

If we stick with Gibson's original concept, then one approach is to articulate the 'ecological' approach that is at the base of Gibson's concept of affordances, and to contrast it with the predominant social constructivist approach. This theoretical tension could be uncomfortably productive, as Gibson's approach is (a) not constructivist, and (b) not social, though it is, in its own way, interactionist. The technology changes the 'habitat' in which we live. This new habitat affords new ways of doing things. An articulation of the affordances of this new environment enables us to adapt more effectively to the (new) opportunities, and avoid the (new) pitfall where 'new' here may indicate a significant degree of change that has marked implications for adaptive behaviour. The transition from the natural habitat that concerned Gibson to the technological habitat of ICT supported learning provides ample opportunities for theoretical elaboration. 
Question 4: The approach suggested reflects a particular interpretation of social theory. Is this a limitation and might a broader analysis of other social theories yield new insights?

If the term is going to be used in a post-Gibsonian way to focus on social factors, which could be productive, then there is an existing relevant body of work (e.g. Laurillard et al., 2000; Kreijns \& Kirschner, 2001; Kreijns et al., 2002). Kreijns et al. (2002), for example, have proposed a theoretical framework for 'social affordances' by suggesting the incorporation of certain properties in Computer Supported Collaborative Leaning (CSCL) environments. These properties are meant to act as social contextual facilitators, i.e. social affordances, to initiate and sustain learner's social interactions. Ultimately these properties are intended to create a social space amongst the members of distributed learning groups. Kreijns et al. (2002) base their environment on a theoretical framework that suggests the embedding of certain properties in CSCL environments:

that act as social-contextual facilitators relevant for the learner's social interactions. When they are perceptible, they invite the learner to act in accordance with the perceived affordances, i.e., start a task or non-task related interaction or communication. (Kreijns et al., 2002)

Furthermore Kreijns et al. (2002) include a software tool that aims to provide the learner group with awareness about the others in the task and in the non-task context. To merely provide affordances for group interaction may be insufficient for the development of a true knowledge-building community. Hewitt et al. (1997) have suggest that a set of tools that permit the individual to monitor communal activity and their own participation in the activity is also required. This a productive direction that is currently being explored by Garnett and Cook (submitted). However, we note that the authors briefly mention as a footnote a planned DialogPlus tool.

Question 5: Practitioners are still exploring the potential of new technologies, and the current uses of technologies often do not take full advantage of the medium. Therefore, how can practice take full advantage of the affordances of ICT?

and

Question 6: Does understanding of the affordances actually get us closer to improvement in practice and is this a useful framework?

Finally, we briefly comment on the potential limits in the scope of the application of this concept to e-Learning and present an alternative. Developing taxonomies that make affordances explicit so that practitioners can make informed choice about existing technology may prove useful. However, perhaps another productive goal would be to develop a framework that allows us to envisage new tools for learning and to describe the communicative contexts in which these tools could be used. For example, Garnett and Cook (submitted) envisage the provision of various computerbased tools to encourage learners to frame their activities in light of a problem (rather 
than a topic), e.g. a tool to create discussion notes; a tool to author with (e.g. to develop a personal web page), a tool to structure learners' interactions in light of statements of proposed problem solutions and information needed to advance that solution. Located at the heart of these learning tools is a Community Knowledge Map. This map would be used to identify hot-spots of community activity and clusters of related work, and to depict the work of the entire community rather than the work of an individual author.

What is required in all approaches is serious attempts to include new empirical observations of learning in the evolution of new frameworks, tools and systems to support (i) tutors as they make decisions about the use of new technology, (ii) learners as they learn, and (iii) systems designers and developers as they envisage new innovative tools to support learning.

\section{Conclusion}

Conole and Dyke have provided a useful examination of the use of affordances to provide a theoretical basis for ICT learning support. We hope some of the issues raised above lead to a productive debate in this area.

\section{References}

Garnett, F. \& Cook, J. (submitted) Models of learning with ICTs in community settings, Technology, Pedagogy and Education.

Gibson, J. J. (1977) The theory of affordance, in: R. Shaw \& J. Bransford (Eds) Perceiving, acting and knowing (Hillsdale, NJ, Erlbaum).

Gibson, J. J. (1979) The ecological approach to visual perception (Hillsdale, NJ, Lawrence Erlbaum).

Hewitt, J., Scardamalia, M. \& Webb, J. (1997) Situative design issues for interactive learning environments: the problem of group coherence, paper presented at the Annual Meeting of the AERA, April.

Laurillard, D., Stratfold, M., Luckin, R., Plowman, L. \& Taylor, J. (2000) Affordances for learning in a non-linear narrative medium, fournal of Interactive Media in Education, 2. Available online at: www-jime.open.ac.uk/00/2

Kreijns, K. \& Kirschner, P. A. (2001) The social affordance of computer-supported collaborative learning environments, $31^{\text {st }}$ ASEE/IEEE Frontiers in Education Conference, 10-13 October, Reno.

Kreijns, K., Kirschner, P. A. \& Jochems, W. (2002) The sociability of computer-supported collaborative learning environments, Educational Technology \& Society, 5(1). Available online at: http://ifets.ieee.org/periodical/vol_1_2002/kreijns.html

McGrenere, J. \& Ho, W. (2000) Affordances: clarifying and evolving a concept, Proceedings of Graphics Interface, May, Montreal. Available online at: http://www.dgp.utoronto.ca/ joanna/ papers/gi_2000_affordances.pdf

Norman, D. (1988) The psychology of everyday things (London, Basic Books).

Norman, D. (1998) The invisible computer (Boston, MIT Press).

Warren (1995) Constructing an econiche, in: P. Flach, J. Hancock, J. Caird \& K. J. Vicente (Eds) Global perspectives on the ecology of human-machine systems (Hillsdale, NJ, Lawrence Erlbaum). 